\title{
A Molecular Perspective of Clinical Benefit: Why Shouldn't Luminal-A Breast Cancer Patients be Considered in Pathological Complete Response Discussions
}

ISSN: 2637-773X

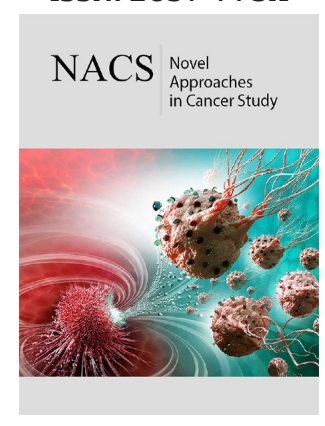

*Corresponding author: Thomas Kieber-

Emmons, tke@uams.edu

Submission: 皆 May 07, 2019

Published: 梅 May 16, 2019

Volume 2 - Issue 5

How to cite this article:Makhoul I, Kieber Emmons T. A Molecular Perspective of Clinical Benefit: Why Shouldn't LuminalA Breast Cancer Patients be Considered in Pathological Complete Response Discussions. Nov Appro in Can Study. 2(5) NACS.000546.2019.

DOI: 10.31031/NACS.2019.02.000546

Copyright@ Kieber Emmons T, This article is distributed under the terms of the Creative Commons Attribution 4.0 International License, which permits unrestricted use and redistribution provided that the original author and source are credited.

\author{
Issam Makhoul ${ }^{1}$ and Thomas Kieber-Emmons ${ }^{2 *}$ \\ Departments of ${ }^{1}$ Medicine and ${ }^{2}$ Pathology, University of Arkansas for Medical Sciences \\ and Winthrop P. Rockefeller Cancer Institute, Little Rock, AR. USA \\ tke@uams.edu
}

\begin{abstract}
Breast cancer is a heterogeneous disease with biological subtypes having different prognostic impacts. This facet is particularly evident in Neoadjuvant trials where Pathological Complete Response (pCR) rates vary. Studies have shown that patients who achieve pCR after neoadjuvant treatment have a better long-term outcome. Consequently, increasing the rate of pCR became the end point of neoadjuvant trials with the expectation of translation into improved survival. However, for luminal-A subtype patients this is not the case, as this cohort does not respond to chemotherapy. Here we consider why this might be the case and several approaches that could change the paradigm treating this cohort.
\end{abstract}

\section{Introduction}

Breast cancer is a spectrum of many subtypes with distinct biological features that lead to differences in response patterns to various treatment modalities and clinical outcomes. Gene expression profiling has led to the molecular classification of breast cancer characterized by intrinsic subtypes: basal-like, HER2-positive, luminal-A, and luminal-B [1]. Up until recently, the subtypes were frequently treated as similar entities. However, there are obvious differences in subtype biological and prognostic characteristics. These differences are clearly evident in the neoadjuvant setting with pathological complete response (pCR) rates being the surrogate marker of efficacy to a therapeutic regime [2,3].

Luminal A breast cancer, for example, is the most common subtype of all breast cancers. They express estrogen receptors (ERs) and/or progesterone receptors (PRs) and are negative for human epidermal growth factor receptor 2 (HER2) expressions. Patients with luminal-A breast cancer have a good prognosis; the relapse rate is significantly lower than other subtypes. Recurrence is common in bone, whereas liver, lung and central nervous system metastases occur in less than $10 \%$ of patients and treatment is mainly based on hormoneblocking therapy. However, patients with luminal-A breast cancers are not so sensitive to paclitaxel- and doxorubicin-containing preoperative or neoadjuvant chemotherapy [4]. Consequently, for luminal-A type breast cancers, the addition of chemotherapy to endocrine therapy generally provides little benefit as deemed from their low pCR rate and long-term impact of chemotherapy on the survival of these patients. Why is this? If we were to find a way to sensitize tumor cells to standard chemotherapy against luminal-A breast cancer would pCR for this subpopulation change clinical approaches outcomes?

\section{The Problem}

From a clinical perspective, there is a need to understand the value of identifying the intrinsic subtypes, as well as gene expression-based classifications, beyond clinicopathological variables that can identify those patients that would benefit from particular therapeutics and the biological mechanisms that explains why subtypes respond to therapies the way they do. One conclusion is that the luminal-A subtype is de novo resistant to chemotherapies. Gene expression profiling of luminal-A type cells suggest that the BCL-2 family of proteins are 
upregulated in these cells [5]. BCL-2 proteins play a central role as guardians against apoptosis, helping cancer cells to evade cell death. Yet, paradoxically in breast cancer, BCL-2 family protein expression correlates with favorable prognosis and estrogen receptor (ER) positivity [6].

The modulation of BCL-2 and its relevance to drug resistance is confounding. On the one hand BCL- 2 down regulation leads to paclitaxel resistance supporting a direct role of BCL-2 downregulation in the maintenance of drug-resistance [7]. Down regulation of BCL-2 has also been proposed as the reason for cisplatin resistance [8]. Yet other studies suggest its upregulation is associated with cisplatin resistance [9]. As BCL-2 is overexpressed in $\sim 75 \%$ of breast cancer, there is much effort toward developing inhibitors targeting BCL-2 to enhance vulnerability to therapy in estrogen receptor-positive breast cancer [10]. Another consideration is Focal Adhesion Kinase (FAK) as a target. FAK is a cytoplasmic nonreceptor tyrosine kinase that enables activation by growth factor receptors or integrin's in the tumor's membrane. The overexpression and activation of FAK is correlated with the poor clinical outcome, highlighting FAK as a potential prognostic marker and anticancer target. Over-expression of FAK has been shown to block the caspase-3-mediated apoptosis; conversely, inhibition of FAK leads to apoptosis in cancer cells [11]. Silencing of FAK with siRNA has shown an ability to overcome taxane resistance [12]. Lastly, the immune suppressive effect of FAK is suggested by the induction of high levels of $\mathrm{Ccl} 5$ in FAK-overexpressing tumors, which inhibits antigen-primed cytotoxic CD8+ T cells and recruits Tregs to the tumor bed, and by the reversal of this effect with FAK inhibitors [13]. Such observations suggest alternative strategies via targeting FAK mRNA or FAK-mediated protein-protein interaction/ signal transduction to advance the development of FAK-targeted therapeutics.

\section{Approach to Augment Chemotherapy}

A novel approach to affect FAK function is the targeting of tumor associated carbohydrate antigens (TACA) representative of lactoseries and ganglioside and antigens. The neolactoseries Lewis $\mathrm{Y}(\mathrm{LeY})$ antigen can also influence the biological behavior of a tumor cell as an important composition of integrin $\alpha v, \beta 3$ by some signal pathway. LeY overexpression may be related to changes in the expression of cell cycle-related factors resulting from activation of the ERK/MAPK and PI3K/Akt signaling pathways. The expression of the LeY antigen, integrin $\alpha \mathrm{v}$ and clinical stage of cancers are both independent, drug resistance-related risk factors.

Antibodies directed against LeY inhibit signaling of LeY modified ErbB receptors [14]. Likewise, GD2, integrin, and FAK form a huge molecular complex across the plasma membrane. GD2+ cells can mediate detachment of cells to undergo anoikis through the conformational changes of integrin molecules and subsequent FAK dephosphorylation [15]. Anti-GD2 monoclonals that affect Akt and FAK activation [16].

We have recently shown that antibodies to GD2 and to LeY induced by a carbohydrate mimetic peptide can sensitize luminal-A breast cancer cells to taxanes. These antibodies mediate apoptosis of cancer cells through caspase 3 [17]. In particular we showed that these antibodies can sensitize the human luminal-A cell line ZR-75 to taxanes [17]. ZR-75 cells express LeY [17]and sialic acid [18]. Sialic acid-binding lectin (SBL) has been shown to have growth suppressive effects on ZR-75 and other breast cancer cell lines attributed to the cancer-selective induction of apoptosis [18]. SBL induces p38 mitogen-activated protein kinase (MAPK) and consequently, activates caspase-3/7 in SBL-induced cell death. Tseng et al. [19] reported that SBL could induce cell death in ERpositive breast tumors through down-regulation of BCL-2 and ER. The anti-tumor effect of SBL-treated ER-positive breast tumors is accompanied by the downregulation of ER and BCL-2. Targeting sialic acid moieties using antibodies that function like SBL or using SBL directly might be additive or synergistic with chemotherapies or with targeted therapies. Consequently, targeting TACA on luminal-A cells might control apoptosis and sensitivity through different mechanisms and pathways.

\section{Clinical Outcome}

While pCR is a surrogate endpoint for prognosis in neoadjuvant chemotherapy for breast cancer patients with grade 1 and 2 who reached pCR still did not do well. Practically, this says that killing all cancer cells in the primary tumor bed does not matter, because patients who had pCR did not do better than those who did not reach pCR. In other words, eradicating all primary tumor cells in ER positive grade 1 and 2 breast cancer is not paralleled with eradication of disseminated tumor cells. This discrepancy might be explained by the pattern of dissemination in each cancer type/grade. If it is true that disseminated tumor cells leave the primary tumor bed as early as the in-situ phase and continue doing so during its invasive phase, one might easily understand the potential heterogeneity of disseminated tumor cells (DTCs) spread throughout the body in cancers with long natural history that spans over decades. In these slow-growing tumors the early DTCs are very likely different from the intermediate or late DTCs in their genomic profile and drug sensitivity. Conversely, fast-growing tumors are likely to have some degree of homogeneity between the primary tumor and the DTCs with similar sensitivity/resistance to chemotherapy [20-22]. Hence, eradicating high grade and rapidly growing tumors in the breast may be associated with eradication of all DTCs that carry the same sensitivity to chemotherapy, leading to a strong correlation between pCR and long-term survival in these patients. This is the challenge.

Raising the numbers of pCR for the sake of raising pCR number in low-grade primary tumors is unlikely to increase survival because many DTCs may escape the cell kill that occurred in the primary tumor bed due to their discordant sensitivity profile. Yet, the fact that pCR rates associated with grade 3 tumors and other tumor subtypes can reflect survival leads to a "what if" one can change grade 1 and grade 2 tumor types to be responsive like grade 3 tumor types. Sensitizing tumor cells with tumor associated carbohydrate antigens reactive antibodies that facilitates modulation of FAK function or interrupts survival-signaling 
pathways to enhance the cytotoxicity of standard chemotherapy would provide a new molecular platform for the development of immunotherapeutic strategies effective against solid tumors and might change treatment paradigms associated with breast cancer.

\section{References}

1. Eliyatkin N, Yalcin E, Zengel B, Aktas S, Vardar E (2015) Molecular classification of breast carcinoma: from traditional, old-fashioned way to a new age and a new way. J Breast Health 11(2): 59-66.

2. Pennisi A, Kieber-Emmons T, Makhoul I, Hutchins L (2016) Relevance of pathological complete response after neoadjuvant therapy for breast cancer. Breast Cancer (Auckl) 10: 103-106.

3. Li J, Chen S, Chen C, Di G, Liu G, et al. (2017) Pathological complete response as a surrogate for relapse-free survival in patients with triple negative breast cancer after neoadjuvant chemotherapy. Oncotarget 8(11): 18399-18408.

4. Rouzier R, Perou CM, Symmans WF, Ibrahim N, Cristofanilli M, et al. (2005) Breast cancer molecular subtypes respond differently to preoperative chemotherapy. Clin Cancer Res 11(16): 5678-5685.

5. Williams MM, Cook RS (2015) Bcl-2 family proteins in breast development and cancer: could Mcl-1 targeting overcome therapeutic resistance? Oncotarget 6(6): 3519-3530.

6. Hwang KT, Han W, Kim J, Moon HG, Oh S, et al. (2017) Prognostic influence of BCL2 on molecular subtypes of breast cancer. J Breast Cancer 20(1): 54-64.

7. Ferlini C, Raspaglio G, Mozzetti S, Distefano M, Filippetti F, et al. (2003) Bcl-2 down-regulation is a novel mechanism of paclitaxel resistance. Mol Pharmacol 64(1): 51-58.

8. Kumar Biswas S, Huang J, Persaud S, Basu A (2004) Down-regulation of Bcl-2 is associated with cisplatin resistance in human small cell lung cancer H69 cells. Mol Cancer Ther 3(3): 327-334.

9. Cho HJ, Kim JK, Kim KD, Yoon HK, Cho MY, et al. (2006) Upregulation of Bcl-2 is associated with cisplatin-resistance via inhibition of Bax translocation in human bladder cancer cells. Cancer Lett 237(1): 56-66.

10. Merino D, Lok SW, Visvader JE, Lindeman GJ (2016) Targeting BCL-2 to enhance vulnerability to therapy in estrogen receptor-positive breast cancer. Oncogene 35(15): 1877-1887.

11. Duxbury MS, Ito H, Zinner MJ, Ashley SW, Whang EE (2004) Focal adhesion kinase gene silencing promotes anoikis and suppresses metastasis of human pancreatic adenocarcinoma cells. Surgery 135(5): 555-562.
12. Halder J, Landen CN, Lutgendorf SK, Li Y, Jennings NB, (2005) Focal adhesion kinase silencing augments docetaxel-mediated apoptosis in ovarian cancer cells. Clin Cancer Res 11(24 Pt 1): 8829-8836.

13. Serrels A, Lund T, Serrels B, Byron A, McPherson RC, et al. (2015) Nuclear FAK controls chemokine transcription, Tregs, and evasion of anti-tumor immunity. Cell 163(1):160-173.

14. Klinger M, Farhan H, Just H, Drobny H, Himmler G, (2004) Antibodies directed against Lewis-Y antigen inhibit signaling of Lewis-Y modified ErbB receptors. Cancer Res 64(3):1087-1093.

15. Aixinjueluo W, Furukawa K, Zhang Q, Hamamura K, Tokuda N, et al. (2005) Mechanisms for the apoptosis of small cell lung cancer cells induced by anti-GD2 monoclonal antibodies: roles of anoikis. J Biol Chem 280(33): 29828-29836.

16. Tsao CY, Sabbatino F, Cheung NK, Hsu JC, Villani V, et al. (2015) Antiproliferative and pro-apoptotic activity of GD2 ganglioside-specific monoclonal antibody 3F8 in human melanoma cells. Oncoimmunology 4(8): e1023975.

17. Hutchins LF, Makhoul I, Emanuel PD, Pennisi A, Siegel ER, et al. (2017) Targeting tumor-associated carbohydrate antigens: a phase I study of a carbohydrate mimetic-peptide vaccine in stage IV breast cancer subjects. Oncotarget 8(58): 99161-99178.

18. Tatsuta T, Sato S, Sato T, Sugawara S, Suzuki T, et al. (2018) Sialic acidbinding lectin from bullfrog eggs exhibits an anti-tumor effect against breast cancer cells including triple-negative phenotype cells. Molecules 23(10): pii: E2714.

19. Tseng HH, Yu YL, Chen YL, Chen JH, Chou CL, et al. (2011) RC-RNaseinduced cell death in estrogen receptor positive breast tumors through down-regulation of Bcl-2 and estrogen receptor. Oncol Rep 25(3): 849853.

20. Makhoul I, Atiq M, Alwbari A, Kieber-Emmons T (2018) Breast cancer immunotherapy: An update. Breast Cancer (Auckl) 12: 1178223418774802 .

21. Sanger N, Effenberger KE, Riethdorf S, Van Haasteren V, Gauwerky J, et al. (2011) Disseminated tumor cells in the bone marrow of patients with ductal carcinoma in situ. Int J Cancer 129(10): 2522-2526.

22. Gruber IV, Hartkopf AD, Hahn M, Taran FA, Staebler A, et al. (2016) Relationship between hematogenous tumor cell dissemination and cellular immunity in dcis patients. Anticancer Res 36(5): 2345-2351. 